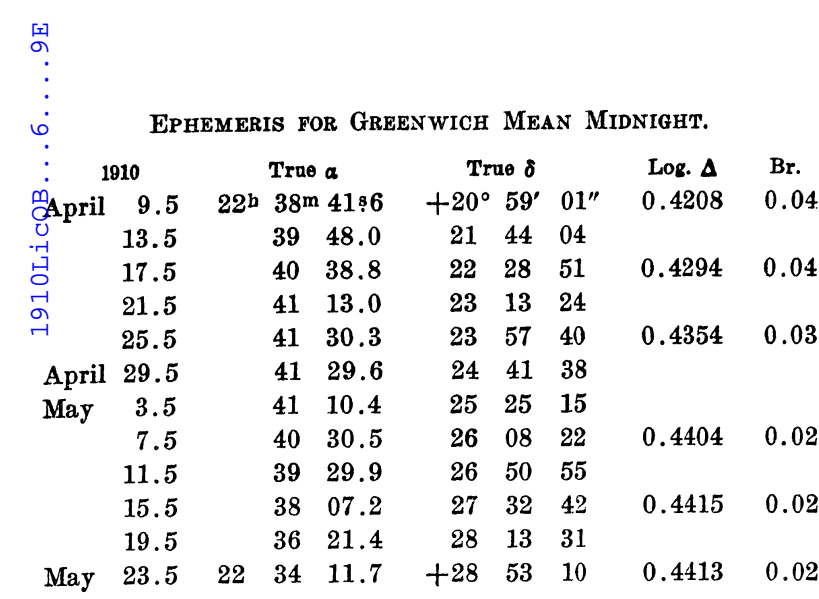

Brightness February $5=1.00$.
The solution of the preliminary and of the final orbit was accomplished without difficulty and without arbitrary variation of the geocentric distance or of any other quantity.

While this bulletin was in press, the following position by Aitken was received by telegraph :

1910 April 12.9998 Gr. M.T., a $22^{\text {h }} 39 \mathrm{~m} 39$ s. 5 , $+21^{\circ} 38^{\prime} 23$ "'

According to this observation the correction to the ephemeris on April 13.0 was $\cos \delta \Delta a=-0$ s. $\Delta \delta=$ $-4^{\prime \prime}$.

W. F. MEYer, Sophia H. Levy.

Berkeley Astronomical Department, April 8, 1910.

\title{
ELLIPTIC ELEMENTS OF COMET e 1909 (DANIEL).
}

In illustration of Professor Leuschner's short methods of computing orbits from three observations, we have computed two orbits for Comet $e \mathbf{1 9 0 9}$ (Daniel), the one from a moderate, the other from a long arc. The first orbit is based on the following observations by Aitken, which were kindly communicated by mail :

\begin{tabular}{|c|c|c|c|c|c|c|}
\hline 1909 Gr. M.T. & & & Comet & Appa & & \\
\hline & & $a$ & & & & \\
\hline & $6^{\mathrm{h}}$ & $17^{\mathrm{m}}$ & $39 \leqslant 13$ & $+38^{\circ}$ & $06^{\prime}$ & $15 "$ \\
\hline & & 18 & 04.46 & +41 & 12 & 21.0 \\
\hline & 6 & 18 & 07.80 & +43 & 26 & 00. \\
\hline
\end{tabular}

It will be observed that the geocentric motion is unfavorable to an accurate derivation of the required initial velocities and accelerations in right ascension and declination when the third and higher differences are neglected so that this example furnishes a good test of the convergency of the formulae.

The direct solution, first approximation, for a parabola resulted in the following residuals:

$$
\begin{array}{rrr}
\text { O-C } & \text { I } & \text { III } \\
\cos \delta \Delta a & +26.7 & +0.1 \\
\Delta \delta & +631.8 & +350.5
\end{array}
$$

Professor Leuschner has shown in his investigations, which are soon to be published, that the middle place and the initial velocities and accelerations can always be represented by a parabola, no matter how erroneous the latter may be, but that residuals will appear in the first and third places, either if the initial velocities and accelerations are inaccurate or if the orbit is not parabolic. It is possible, in general, by the application of a simple criterion, to decide at this stage of the computation to which of these two causes the residuals are to be ascribed and to complete the solution of the elements without hypothesis regarding the eccentricity, if the residuals are not found to be wholly due to the neglect of third and higher differ- ences in deriving the initial velocities and accelerations from the three observations.

Although in the present case the criterion clearly established the periodicity of the orbit, it was evident that the residuals were due in part to the neglect of the third and higher differences of the observed coördinates. The computation of the elements was therefore not immediately completed without hypothesis regarding the eccentricity, but a very approximate differential correction of the parabolic geocentric distance and of the heliocentric velocities at the middle date was undertaken so as to reduce the residuals, on the one hand with, and on the other without hypothesis regarding the eccentricity. This double solution was readily accomplished on account of the convenience with which it is possible in the short methods to pass from one class of orbit to another. The resulting residuals were :

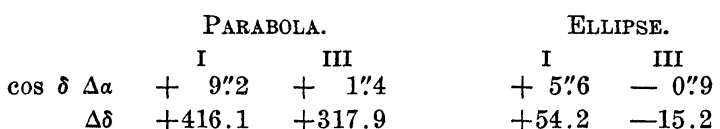

These residuals confirm the conclusion arrived at by the criterion referred to above, that the observations cannot be satisfied by a parabola. The removal of the residuals for the ellipse by a single application of the differential formulae produced the following elements :

$$
\begin{aligned}
& \text { Elements. } \\
& T=1909 \text { November } 28.1924 \text { Gr. M.T. } \\
& \omega=03^{\circ} \quad 02^{\prime} \quad 011^{\prime \prime} 7 \text { ) }
\end{aligned}
$$

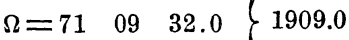

$$
\begin{aligned}
& i=19 \quad 54 \quad 39.0\} \\
& e=0.62524 \\
& \mu=496 \text { ". } 1411 \\
& \log a=0.569601 \\
& \text { Period }=7.15157 \text { years }
\end{aligned}
$$


The publication of these elements was deferred so that they might be tested and improved on the basis of later observations. In the meantime they were verified by the results published by Ebell in A.N. 4384 .

The long are orbit is based on the following observations :

Dec. $\quad 7.66050$

Dec. 18.75589

$6^{\mathrm{h}} 16^{\mathrm{m}} 42 \mathrm{~s} 10+34^{\circ} 44^{\prime} 22^{\prime \prime} 0$

$\begin{array}{llllll}6 & 18 & 07.80 & 43 & 26 & 00.0\end{array}$

Barnard (Yerkes)

March 3.47726

$\begin{array}{lllll}7 & 25 & 03.4 \quad+52 \quad 54 \quad 42\end{array}$

Aitken

Rambaud (Algiers)

The first and third of these positions are represented as follows on the basis of the first orbit:

$$
\begin{array}{ccc}
\text { O-C } & \text { I } & \text { III } \\
\cos \delta \Delta \alpha & -1.5 & +319^{\prime \prime} 4 \\
\Delta \delta & +9.3 & -735.8
\end{array}
$$

The application of the differential formulae on the basis of closed expressions for $\delta f$ and $\delta g$ so as to remove the residuals resulted in

$$
\begin{array}{cccc}
\text { O-C } & & \text { I } & \text { III } \\
\cos \delta & \Delta a & -0.7 & +3.6 \\
& \Delta \delta & -0.7 & -11.7
\end{array}
$$

Without recomputation of the differential coefficients the substitution of these residuals in the absolute terms of the equations produced the following results, which represent the three given positions:

ELEMENTS.

$T=1909$ November 28.7238 Gr. M.T. $\omega=03^{\circ} 28^{\prime} \quad 43.9$

$\Omega=70 \quad 59 \quad 43.4 \quad 1910.0$

$i=19 \quad 26 \quad 48.1\}$

$e=0.602481$

$\mu=547^{\prime \prime} 5362$

$\log a=0.541063$

Period $=6.48030$ years

Constants For the Equator 1910.0

$$
\begin{aligned}
& x=r[9.977341] \sin \left(163^{\circ} 24^{\prime} 50.1+v\right) \\
& y=r[9.944549] \sin \left(\begin{array}{llll}
83 & 43 & 06.4+v
\end{array}\right) \\
& z=r[9.755574] \sin \left(\begin{array}{llll}
44 & 49 & 24.1 & +v
\end{array}\right)
\end{aligned}
$$

Sturla Einarsson, R. YounG.

Berkeley Astronomical Department, April 8, 1910. 